\title{
The mitokine quest(ion)
}

\author{
Cell Research (2016) 26:1265-1266. doi:10.1038/cr.2016.138; published online 25 November 2016
}

Cells and organisms adapt to mitochondrial dysfunction by activating the mitochondrial unfolded protein response $\left(U P R^{\mathrm{mt}}\right)$, which is regulated by mitochondrial-to-nuclear communication; and $U P R^{\mathrm{mt}}$ activation can also be transmitted between different cell types suggesting a role in tissue coordination. Shao and colleagues now identify a neuronal circuit and a secreted neuropeptide required for cell non-autonomous $\mathrm{UPR}^{\mathrm{mt}}$ regulation.

Mitochondrial dysfunction can originate from genetic lesions or pathogenproduced toxins that impair mitochondrial function (oxidative phosphorylation, etc.), as well as by proteotoxic effects within the organelle. One response employed to alleviate mitochondrial stress is the $\mathrm{UPR}^{\mathrm{mt}}$, a transcriptional response of over 500 transcripts that promotes the repair and recovery of defective mitochondria, metabolic adaptations, xenobiotic detoxification and innate immunity. In C. elegans, the UPR ${ }^{\mathrm{mt}}$ is regulated by the transcription factor ATFS-1 [1] coupled with extensive chromatin remodeling [2]. ATFS-1 activity is regulated by organelle partitioning and mitochondrial protein import efficiency. ATFS-1 is expressed in all tissues and imported into healthy mitochondria where it is degraded. However, if mitochondrial function is perturbed, protein import efficiency is reduced, causing ATFS-1 to accumulate in the cytosol. Because ATFS-1 also has a nuclear localization sequence, it then traffics to the nucleus to activate the transcriptional response.

In addition to intracellular signaling, $\mathrm{UPR}^{\mathrm{mt}}$ activation within neurons can be communicated to distal tissues such as the intestine to activate the response in these cells. Non-autonomous UPR ${ }^{\mathrm{mt}}$ activation likely promotes coordination of mitochondrial function or metabolic adaptations across tissues, which may contribute to the metabolic abnormalities found in distal tissues in neurodegenerative diseases such as Huntington's disease and Parkinson's disease [3]. Because neurons do not innervate the worm intestine, non-automonous UPR ${ }^{\mathrm{mt}}$ signaling has been hypothesized to be mediated by a "mitokine" [4], however, the mode of signal transduction has remained unclear, as have the functional ramifications.

Using an impressive variety of cell type-specific CRISPR techniques, Shao and colleagues now demonstrate that multiple forms mitochondrial dysfunction (impairment of a mitochondrial protease, the respiratory chain, localized reactive oxygen species or depletion of inner membrane potential) administered specifically within neurons are capable of stimulating UPR $^{\mathrm{mt}}$ activation in neurons [5]. Interestingly, all of the neuronal mitochondrial stresses other than membrane potential depletion caused subsequent intestinal cell UPR ${ }^{\mathrm{mt}}$ activation suggesting that not all forms of mitochondrial dysfunction activate the non-autonomous $\mathrm{UPR}^{\mathrm{mt}}$. The robust system was used to identify a sub-neuronal circuit required for propagation of the UPR ${ }^{\mathrm{mt}}$ between tissues. The neural circuit required three sensory neurons capable of sampling the environment and the AIA interneuron, which receives and integrates information from sensory neurons for downstream signaling. Because the interneuron does not physically interact with the intestine, the authors considered secreted signaling molecules such as neuropeptides. The requirement for a neuropeptide processing protease for non-autonomous
$\mathrm{UPR}^{\mathrm{mt}}$ induction strongly supported the involvement of at least one of the $\sim 250$ known neuropeptide(s) in C. elegans.

An elegant neuron-specific CRISPR screen identified six neuropeptides required for UPR ${ }^{\mathrm{mt}}$ propagation across tissues, but only one of these was sufficient. Similar to neuron-specific activation of ATFS-1, neuron-specific expression of FLP-2 was sufficient to induce the UPR ${ }^{\mathrm{mt}}$ in the intestine. Further, deletion of $f l p-2$ within a single interneuron impaired nonautonomous UPR ${ }^{\mathrm{mt}}$ activation initiated within the sensory neurons. While the $\mathrm{UPR}^{\mathrm{mt}}$-mitokine story is not complete, the discovery of a sub-neuronal circuit and a secreted neuropeptide by Shao and colleagues represents a significant step forward allowing the field to focus on events downstream of the interneuron and FLP-2 secretion.

FLP-2 may well be a peptide secreted during mitochondrial stress to affect other cell types, which fits the definition of a mitokine. However, those cell types and the receptor remain to be identified as expression of FLP-2 specifically by intestinal cells was not sufficient to activate the intestinal $\mathrm{UPR}^{\mathrm{mt}}$ [5]. Thus, FLP-2 likely functions elsewhere. Similarly, a recent study demonstrated a requirement for the neuronal release of serotonin in activation of the non-autonomous UPR ${ }^{\mathrm{mt}}$, but how serotonin effects intestinal cells remains to be determined [3]. While the relationship between FLP-2 and serotonin has not been examined, it is safe to assume that a number of interesting signaling events occur downstream of both molecules to affect the regulation of intestinal ATFS-1. Both studies indicate that ATFS-1 is essential in both the sensing neurons as well as in the intestinal cells for non-autonomous UPR ${ }^{\mathrm{mt}}$ activa- 


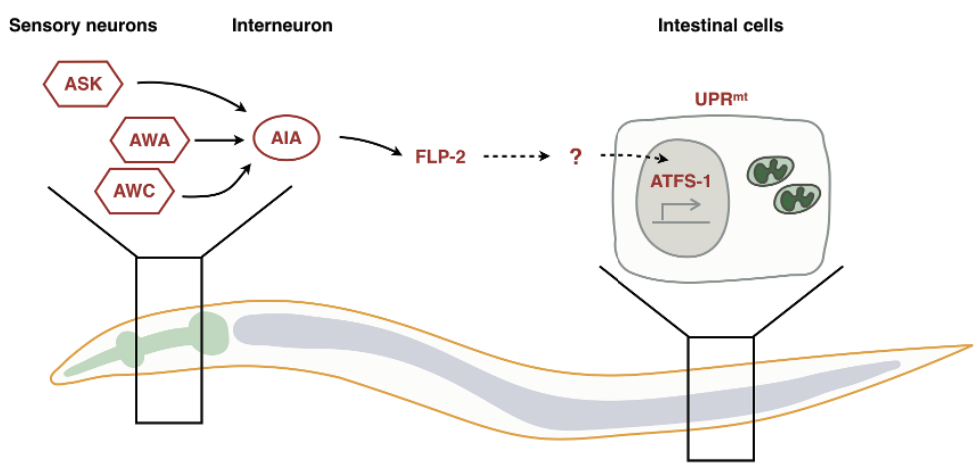

Figure 1 Cell non-autonomous regulation of the UPR ${ }^{\mathrm{mt}}$. Mitochondrial perturbations within three $C$. elegans sensory neurons (ASK, AWA, AWC) causes activation of ATFS-1 and the UPR ${ }^{\mathrm{mt}}$ within these cell types and also within intestinal cells. Transmission of a signal between the sensory neurons requires an interneuron (AIA), the secreted neuropeptide FLP-2 as well as ATFS-1 within the intestinal cells. It is currently unclear how secreted FLP-2 affects intestinal ATFS-1.

tion $[3,5]$. In neurons where mitochondria are perturbed directly, ATFS-1 activation is likely achieved by impaired mitochondrial protein import efficiency. But, how is ATFS-1 activated in the receiving intestinal cells? Does non-autonomous signaling perturb mitochondrial function within the intestine or somehow engage ATFS-1 independent of mitochondrial dysfunction? Alternatively, non- autonomous signaling may promote chromatin rearrangements in intestinal cells, which has been shown to activate ATFS-1 and the $\mathrm{UPR}^{\mathrm{mt}}$ [2].

It will also be important to determine the functional effects of non-autonomous $\mathrm{UPR}^{\mathrm{mt}}$ signaling. Stated differently, in what physiologic scenarios do sensory neurons first encounter mitochondrial stress and what benefit is conferred by $\mathrm{UPR}^{\mathrm{mt}}$ activation in distal tissues? The authors suggest a role in sensing mitochondrial stress, which may propagate a signal to distal tissues to "pre"-activate or prime a response. Given the role of the $\mathrm{UPR}^{\mathrm{mt}}$ in innate immunity and responding to pathogens that produce mitochondrial toxins, this may provide a means to activate the UPR ${ }^{\mathrm{mt}}$ in intestinal cells before the pathogen has accumulated to a level within the intestinal lumen that severely perturbs mitochondrial function [6,7]. Alternatively, because $\mathrm{UPR}^{\mathrm{mt}}$ activation shifts metabolism from oxidative phosphorylation to glycolysis to facilitate mitochondrial repair [7], non-autonomous signaling may simply coordinate metabolism between different tissues or organs. Consistent with this idea, the mammalian hormone Fgf21 has been suggested to be a mitokine as its secretion is induced during mitochondrial stress via the stress-activated transcription factor ATF4. In this context, Fgf21 produced by muscle cells promotes mitochondrial biogenesis and fat browning in adipocytes along with enhanced lipid catabolism [8].

\section{Pan Deng ${ }^{1,2}$, Cole M Haynes ${ }^{1,2}$ \\ ${ }^{1}$ University of Massachusetts Medical School, De- partment of Molecular, Cell and Cancer Biology, Lazare Medical Research Building, Worcester, MA 01605, USA, ${ }^{2}$ Weill Cornell Medical College, BCMB Allied Program, New York, NY 10065, USA Correspondence: Cole M Haynes \\ E-mail: cole.haynes@umassmed.edu}

\section{References}

1 Nargund AM, Pellegrino MW, Fiorese CJ, et al. Science 2012; 337:587-590.

2 Tian Y, Garcia G, Bian Q, et al. Cell 2016; 165:1197-1208.

3 Berendzen KM, Durieux J, Shao LW, et al. Cell 2016; 166:1553-1563.

4 Durieux J, Wolff S, Dillin A. Cell 2011; 144:79-91.

5 Shao LW, Niu R, Liu Y. Cell Res 2016; 26:1182-1196.

6 Liu Y, Samuel BS, Breen PC, et al. Nature 2014; 508:406-410.

7 Pellegrino MW, Nargund AM, Kirienko NV, et al. Nature 2014; 516:414-417.

8 Lin YF, Haynes CM. Mol Cell 2016; 61:677682.

9 Kim KH, Jeong YT, Oh H, et al. Nat Med 2013; 19:83-92. 